BULL. AUSTRAL. MATH. SOC.

MOS 0540,0525

VOL. I (1969), 325-331.

\title{
Certain graphs arising from Hadamard matrices
}

\author{
W. D. Wallis
}

\begin{abstract}
We establish several infinite classes of regular graphs with the property that any two distinct vertices have a fixed number of other vertices joined to both of them. The graphs are found by constructing their incidence matrices, which correspond to certain Hadamard matrices.
\end{abstract}

\section{Preliminaries}

We assume such standard ideas as $(v, k, \lambda)$ - configuration and Hadamard matrix (see, for example, [2]). By a $(v, k, \lambda)$-graph $G$ we mean a regular graph with $v$ points, of valency $k$, such that for any pair of points there are exactly $\lambda$ points joined to both by ares of $G$. In other words, if $B_{i}$ is the set of all points joined to point $i$, then

$$
\begin{aligned}
& B_{i} \text { has } k \text { elements for every } i ; \\
& B_{i} \cap B_{j} \text { has } \lambda \text { elements whenever } i \neq j .
\end{aligned}
$$

It is clear that $B_{1}, \ldots, B_{v}$ are the blocks of a $(v, k, \lambda)$-configuration whose varieties are the points of $G$; this configuration has the properties

$$
\begin{aligned}
& i \notin B_{i} \text { for any } i ; \\
& i \in B_{j} \Leftrightarrow j \in B_{i} .
\end{aligned}
$$

On the other hand, if we have such a configuration, we can form a graph by "join points $i$ and $j$ when $i \in B_{j}$ ". Recasting this in terms of the incidence matrix of the configurataion, we have the following

Received 12 May 1969. 
characterization:

(1) There is a $(v, k, \lambda)$-graph if and only if there is a $(v, k, \lambda)$-configuration whose incidence matrix is symmetric and has diagonal $(0,0, \ldots, 0)$.

Consequently the necessary conditions for the existence of a $(v, k, \lambda)$-configuration $[2, p .107]$ :

(2) $\lambda(v-1)=k(k-1)$;

(3) (i) if $v$ is even then $(k-\lambda)$ is a perfect square,

(ii) if $v$ is odd then

$$
z^{2}=(k-\lambda) x^{2}+(-1)^{(v-1) / 2} \lambda y^{2}
$$

has a non-trivial integer solution;

are also necessary for a $(v, k, \lambda)$-graph.

Ahrens and Szekeres [1] have proposed the question: for what triples $(v, k, \lambda)$ is there a $(v, k, \lambda)$-graph? We shall establish the existence of certain infinite classes of graphs.

I would like to thank Mr Ahrens and Professor Szekeres for their helpful correspondence, and for sending me a prepublication copy of [1].

\section{Correspondence between graphs and Hadamard matrices}

It is known [2, p. 206] that if there is a $\left(4 u^{2}, 2 u^{2}-u, u^{2}-u\right)$-configuration with incidence matrix $A$, then there is an Hadamard matrix $E$ of order $4 u^{2}$,

$$
H=J-2 A,
$$

where $J$ is the matrix with every element +1 . On the other hand, suppose $H$ is an Hadamard matrix of order $4 u^{2}$ with exactly $2 u^{2}-u$ elements -1 in each row. Then $H J=J H^{T}=2 u J$; so if $A=\frac{1}{2}(J-H)$,

$$
\text { AA } \begin{aligned}
T & =\frac{1}{4}\left(4 u^{2} J-H J-J H^{T}+4 u^{2} I\right) \\
& =u^{2} I+\left(u^{2}-u\right) J .
\end{aligned}
$$

$A$ is a $(0,1)$-matrix, so it is the incidence matrix of a $\left(4 u^{2}, 2 u^{2}-u, u^{2}-u\right)$-configuration. The $I^{\prime} s$ in $A$ are in the same positions 
as the -1 's in $H$. Therefore, if $H$ is symmetric and has +1 in every diagonal position, $A$ will be the incidence matrix corresponding in ( 1 ) to a $\left(4 u^{2}, 2 u^{2}-u, u^{2}-u\right)-g r a p h$. We will refer to a graph with these parameters as being "of type $A_{u}$ "; we have proven

THEOREM 1. There is a graph of type $A_{u}$ if and only if there is a symmetric Hadamard matrix of order $4 u^{2}$, with diagonal elements all +1 and with exactly $2 u^{2}+u$ elements +1 in every row.

We will call a $\left(4 u^{2}, 2 u^{2}+u, u^{2}+u\right)$-graph one "of type $B_{u}^{\prime \prime}$. Analogously to the above, we can prove

THEOREM 2. There is a groph of type $B_{u}$ if and only if there is a symmetric Hadamard matrix of order $4 u^{2}$, with diagonal elements all +1 and with exactly $2 u^{2}-u$ elements +1 in every row.

Another well-known correspondence between configurations and Hadamard matrices is as follows: suppose $A$ is the incidence matrix of a $(4 n-1,2 n, n)$-configuration. Construct a matrix $B$ by replacing every 0 of $A$ by +1 and every 1 of $A$ by -1 . Then put

$$
B=\left[\begin{array}{c|ccc}
1 & 1 & \ldots & 1 \\
\hline 1 & & \\
\vdots & & B
\end{array}\right] .
$$

$H$ is Hadamard of order $4 n$. Conversely, if $H$ is a Hadamard matrix whose first row and column consist of entries +1 , the process can be reversed to produce the incidence matrix of a $(4 n-1,2 n, n)$-configuration. If, further, $H$ has diagonal entries all +1 and is symmetric, then by (1) the configuration corresponds to a $(4 n-1,2 n, n)$-graph. We say a graph is "of type $c_{u}^{\prime \prime}$ if it is a $\left(4 u^{2}-1,2 u^{2}, u^{2}\right)-g r a p h$.

THEOREM 3. There is a graph of type $c_{u}$ if and only if there is a symmetric Hadamard matrix of order $4 u^{2}$ with diagonal entries all +1 .

This follows from the preceding remarks.

THEOREM 4. If there is a graph of type $A_{u}$ or a graph of type $B_{u}$ then there is a groph of type $c_{u}$. 
Proof. Suppose there is a graph of type $A_{u}$. Then by Theorem 1 there is a symmetric Hadamard matrix $A$ with all diagonal entries +1 and with exactly $2 u^{2}+u$ entries +1 in every row. Negate all rows of $A$ with first element -1 , and negate the corresponding columns. The resulting matrix $C$ is Hadamard, is symmetric, has every diagonal entry +1 and has every entry in the first row and column +1 . The existence of $C$ together with Theorem 3 imply the existence of a graph of type $k_{u}$. If we assume the existence of a $B_{u}$, a similar proof applies.

\section{Kronecker products}

Given two matrices $A$ and $B$, where $A$ is $p \times q$, the Kronecker product of $A$ and $B$ is

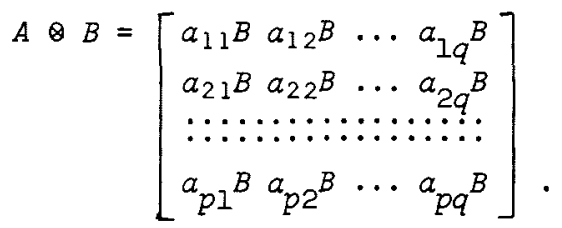

It is well-known that if $A$ and $B$ are Hadamard then so is $A \otimes B$.

THEOREM 5. If there are graphs of types $A_{u}$ and $B_{v}$, or there are graphs of types $B_{u}$ and $A_{v}$, then there is a graph of type $B_{2 u v}$.

Proof. Suppose $A$ and $B$ are symmetric Hadamard matrices corresponding to graphs of types $A_{u}$ and $B_{v}$ respectively. Then $A \otimes B$ is a symmetric Hadamard matrix of order $16 u^{2} v^{2}$;

$A$ and $B$ have all diagonal entries +1 , so $A \otimes B$ has the same; $A$ has exactly $2 u^{2}+u$ elements +1 and $2 u^{2}-u$ elements -1 per row ;

$B$ has $2 v^{2}-v$ positive and $2 v^{2}+v$ negative, so the number of entries +1 in any row of $A \otimes B$ is

$$
\begin{aligned}
\left(2 u^{2}+u\right)\left(2 v^{2}-v\right)+\left(2 u^{2}-u\right)\left(2 v^{2}+v\right) & =8 u^{2} v^{2}-2 u v \\
& =2(2 u v)^{2}-(2 u v) .
\end{aligned}
$$

Therefore (using Theorem 2) $A \otimes B$ corresponds to a graph of type $B_{2 u v}$. If we assume that there are graphs of types $B_{u}$ and $A_{v}$, the proof is 
similar.

In the same way we can prove

THEOREM 6. If there are graphs of types $A_{u}$ and $A_{v}$, or there are graphs of types $B_{u}$ and $B_{v}$, then there is a graph of type $A_{\text {auv }}$.

If $A$ and $B$ are symmetric Hadamard matrices of order $4 u^{2}$ and $4 v^{2}$ with every diagonal entry +1 , then $A \otimes B$ will be a matrix of order $16 u^{2} v^{2}$ with the same properties. Therefore

THEOREM 7. If there are graphs of types $c_{u}$ and $c_{v}$, then there is a graph of type $c_{2 u v}$.

\section{Some particular graphs.}

In order to use Theorems 5, 6 and 7, we need to show that at least some $(v, k, \lambda)$-graphs of the various types exist. In fact there are graphs of types $A_{1}, B_{1}, C_{1}, A_{3}, B_{3}, C_{3}, C_{5}$ and $C_{7}$.

For any $n$ it is clear that the complete graph of order $n$ is an $(n, n-1, n-2)$-graph. For example, the $(3,2,1)$ and $(4,3,2)$ graphs exist:
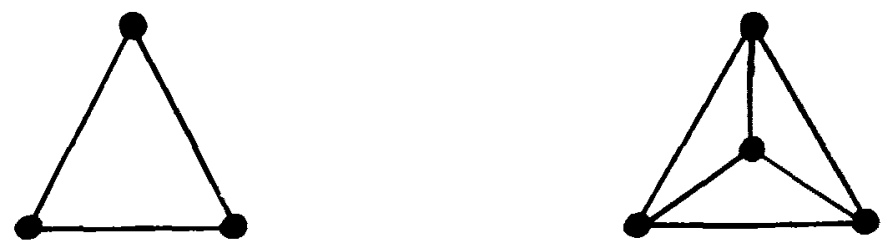

These are graphs of types $C_{1}$ and $B_{1}$ respectively; their incidence matrices are

$$
\left[\begin{array}{lll}
0 & 1 & 1 \\
1 & 0 & 1 \\
1 & 1 & 0
\end{array}\right] \quad\left[\begin{array}{llll}
0 & 1 & 1 & 1 \\
1 & 0 & 1 & 1 \\
1 & 1 & 0 & 1 \\
1 & 1 & 1 & 0
\end{array}\right]
$$

and the corresponding Hadamard matrices are 


$$
\left[\begin{array}{rrrr}
1 & 1 & 1 & 1 \\
1 & 1 & -1 & -1 \\
1 & -1 & 1 & -1 \\
1 & -1 & -1 & 1
\end{array}\right],\left[\begin{array}{rrrr}
1 & -1 & -1 & -1 \\
-1 & 1 & -1 & -1 \\
-1 & -1 & 1 & -1 \\
-1 & -1 & -1 & 1
\end{array}\right] \text {. }
$$

If $n$ is even, then we can form an $(n, 1,0)$ graph by joining points 1 to 2,3 to 4 , and so on: the pairs joined by the arcs are the pairs $\{2 i-1,2 i\}$. The $(4,1,0)$ and $(6,1,0)$ graphs are
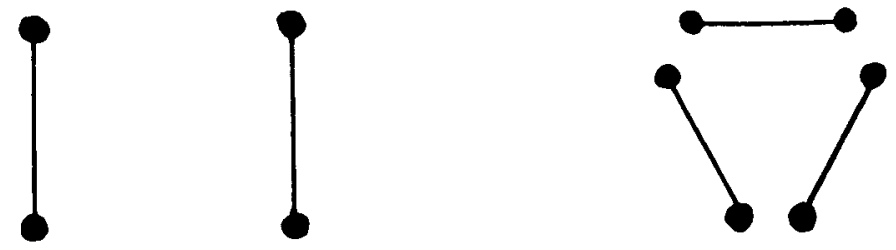

The first of these is of type $A_{l} ;$ its incidence matrix and Hadamard matrix are respectively

$$
\left[\begin{array}{llll}
0 & 1 & 0 & 0 \\
1 & 0 & 0 & 0 \\
0 & 0 & 0 & 1 \\
0 & 0 & 1 & 0
\end{array}\right],\left[\begin{array}{rrrr}
1 & -1 & 1 & 1 \\
-1 & 1 & 1 & 1 \\
1 & 1 & 1 & -1 \\
1 & 1 & -1 & 1
\end{array}\right] \text {. }
$$

In [3] there is exhibited the incidence matrix of a $(36,15,6)$-configuration. The matrix is symmetric and has zero diagonal, so it corresponds to a $(36,15,6)$-graph; therefore there is a graph of class $A_{3}$. In this particular matrix, if we carry out the operations

(i) for every element in columns 10-27, replace every 0 by a 1 and every 1 by a 0 ,

(ii) for every element in rows 10-27 of the resulting matrix, replace every 0 by a 1 and every 1 by a 0 ,

then the resulting matrix corresponds to a $(36,21,12)-g r a p h$. So there is a graph of type $B_{3}$. By Theorem 4 there is a graph of type $C_{3}$.

R.W. Ahrens (personal communication) has shown that if there is a balanced incomplete block design of parameters

$$
\left(4 u^{2}-1,2 u^{2}-u, 2 u+1, u, 1\right)
$$

then there is a graph of type $\mathcal{C}_{u}$ : the vertices of the graph correspond to 
the blocks of the design, and two vertices are joined by an arc if and only if the corresponding blocks have non-empty intersection. Block designs of the required parameters are given in Appendix $I$ of [2] for $u=2,3,4,5$ and 7 , so there are graphs of types $C_{2}, C_{3}, C_{4}, C_{5}$ and $C_{7}$.

Combining these results and using Theorems 5,6 and 7 , we have

THEOREM 8. There are graphs of types $A_{u}$ and $B_{u}$ for any $u$ of the form $2^{a} 3^{b}$, where $a$ and $b$ are non-negative integers such that

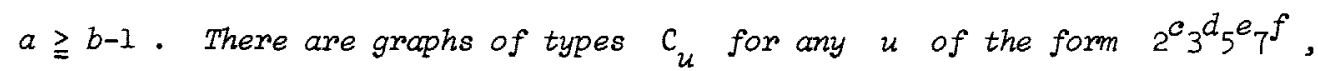
where $c, d, e$, and $f$ are non-negative integers such that $c \geqq d+e+f-1$.

\section{References}

[1] R.W. Ahrens and G. Szekeres, "On a combinatorial generalization of twenty-seven lines associated with a cubic surface", J. Austrat. Math. Soc. (to appear).

[2] Marshall Hall, Jr, Combinatorial theory (Blaisdell, Waltham, Massachusetts, 1967).

[3] Jennifer Wallis, "Two new block designs", J. Combinatorial Theory (to appear).

La Trobe University,

Bundoora, victoria. 